\title{
Promoción DEL MÉTODo DE PURIFICACIÓN DE AGUA PARA USO DOMÉSTICO, EN LA COMUNIDAD de La Tola del GADIP de Cayambe
}

\author{
PROMOTION OF THE METHOD OF PURIFICATION \\ OF WATER FOR DOMESTIC USE, IN THE COMMUNITY \\ of LA Tola OF THE GADIP OF CAYAMBE
}

IVONNE CARRILLO P.

Universidad Internacional SEK

ÁLVARO ZAMBRANO RUILOVA

Universidad Tecnológica Equinoccial

Correo electrónico: alvaro.zambrano@ute.edu.ec

VÍCTOR HUGO ÁRIAS

Universidad Tecnológica Equinoccial

ALEXANDRA ENDARA

FECHA DE RECEPCIÓN: 07/11/2016

Universidad Internacional SEK

FECHA DE ACEPTACIÓN: 16/12/2016

\section{RESUMEN}

La creciente demanda de agua potable para el consumo humano en el Ecuador hace necesario contar con mecanismos de abastecimiento y distribución por parte de los gobiernos seccionales en el país. Desde 1999 en varios países de América Latina, al igual que en África y Asia, se lanzaron iniciativas y actividades locales de aplicación de métodos, entre ellos el método SODIS (Solar DISinfection). El presente proyecto busca mejorar la calidad de vida de la comunidad La Tola perteneciente al Gobierno Autónomo Descentralizado Intercultural y Plurinacional, GADIP, de Cayambe, al tratar el agua para consumo y cumplir el mandato del Artículo 14 de la Constitución de la República sobre "el derecho de la población a vivir en un ambiente sano y ecológicamente equilibrado, que garantice la sostenibilidad y el buen vivir, Sumak Kawsay".

Para tal efecto, apoyados por técnicos de la Asociación de Municipalidades Ecuatorianas, AME, se definió la necesidad de la Comunidad y la posibilidad de implementación del proyecto, para lo que se consolidó el Convenio UTE-GADIP de Cayambe como base legal para incursionar y dar solución al problema puntual de la comunidad con respecto al uso del agua y las prácticas de higiene.

Palabras clave: Calidad de vida, método, purificación del agua. 


\begin{abstract}
The growing demand for drinking water for human consumption in Ecuador makes it necessary to have mechanisms for supply and distribution leaded by the Sectional Governments in the country. Since 1999, in several countries in Latin America, as well as in Africa and Asia, local initiatives and methods have been launched, including the SODIS (Solar Disinfection) method. The present project seeks to improve the quality of life of La Tola community, part of the Autonomous Decentralized Intercultural and Plurinational Government, GADIP, of Cayambe, by treating drinking water for human consumption in order to fulfill the mandate of Article 14 of the Constitution of the Republic on the "right of the population to live in a healthy and ecologically balanced environment that guarantees sustainability and good living, Sumak Kawsay”.

To this end, and supported by technicians of the Association of Ecuadorian Municipalities, AME, we defined the main needs of the Community and the possibility of implementing the project, by signing the Cayambe UTE-GADIP Agreement to consolidate legal basis for entering and giving solution to this problem of the community with respect to water use and hygiene practices.
\end{abstract}

Keywords: Quality of life, method, water purification.

\section{Antecedentes}

La realización de este artículo es consecuencia de haber dirigido un Proyecto de Vinculación con la Colectividad en la Comunidad La Tola del GADIP de Cayambe y que fuera liquidado en marzo de 2016.

La demanda de agua potable en Ecuador genera la necesidad de encontrar soluciones a favor de sus habitantes (RO.305 de 6.Ago.2014). En la Comunidad, fundamentalmente agrícola, sus habitantes cumplen las labores fuera de su población; son los niños, en ciertos casos con sus madres, quienes pasan la mayor parte del tiempo expuestos a los riesgos que puede ocasionar el consumo del agua que se capta únicamente de una vertiente subterránea del Nevado Cayambe, ubicada en el interior de una propiedad privada. Su evaluación resulta complicada, por la serie de autorizaciones requeridas para acceder a esta parte del predio desde donde se distribuye el agua a la población mediante mangueras que no reciben ningún mantenimiento.

El método SODIS, avalado por la AME, es socializado a las autoridades y Comunidad, que al aprovechar la radiación ultravioleta (UV) del sol, reduce la carga microbiana del agua contaminada que se capta de la vertiente natural, a niveles que permiten su consumo según los requisitos (Norma Técnica Ecuatoriana NTE INEN 1108 Agua Potable, requisitos, 2014).

La eficacia del método SODIS se evidenció en pruebas físico-químicas de las muestras en Laboratorios TRAHISA. Previo al uso del método y su posterior aplicación, se realizaron ensayos en los laboratorios de Ingeniería Ambiental de la UTE, utilizando placas petrifilm y los procedimientos requeridos para manejo, toma de muestras y procedimientos de incubación.

\section{Marco teórico}

El agua es la riqueza natural más importante que en el organismo humano representa del 45 al $60 \%$ del peso corporal (CEPAL, 2012) y debe ser consumida en promedio diario ocho vasos y así reponer parte de los tres litros que se pierden en condiciones normales, la otra mitad se recupera ingiriendo alimentos y de la metabolización de los azúcares consumidos. 
Alrededor del 40\% de las comunidades en el planeta no tienen acceso al agua potable (CEPAL, 2012) lo que genera problemas de salud; son muchos estudios y esfuerzos que se desarrollan para dar solución a la carencia de agua tratada de manera efectiva. Este estudio hipotético-deductivo busca mediante el método SODIS inactivar los nocivos microorganismos patógenos (virus, bacterias, parásitos) que causan enfermedades, valiéndose de los dos efectos de la radiación solar, donde la radiación UV y el calor que incide en el agua, producen sinergia y logra que el efecto simultáneo de ambos sea mucho mayor que la suma de cada uno de ellos; (Desinfección Solar del agua, Guía de aplicación, 2003) para tal propósito el sistema de tratamiento del agua dulce debe ser aplicado en localidades donde el efecto del sol tenga una ocurrencia suficiente para que el agua llegue a los $50^{\circ} \mathrm{C}$., expuesta durante al menos 5 a 6 horasen botellas de plástico transparente hechas de tereftalato de polietileno PET. Si el nivel de energía calorífica es menor y la nubosidad es superior al 50\%, es necesario exponer el agua durante dos días como condición para lograr una calidad de agua segura para ser consumida y con una carga bacteriológica lo suficientemente baja.

Desde 1999, existen iniciativas de aplicación deSODIS, en países de América Latina, así como en Indonesia, Sri Lanka, India, Nepal, Pakistán, Kenya, Sudáfrica, Angola, etcétera, a través de organizaciones como la EAWAG/SANDEC que apoya a sus socios locales en la elaboración de material informativo y en el desarrollo de estrategias de promoción, incluidas en campañas de publicidad del método a nivel internacional. Ref: http://www.sodis.ch.

\section{Metodología}

Se plantea a las autoridades de la Facultad iniciativas para intervenir en proyectos de Vinculación con la Comunidad, resultando el Proyecto "Promoción del Método de Purificación de Agua para uso Doméstico", el más viable de aplicar; para el efecto, se generan acuerdos, reuniones de planificación y capacitación a docentes y estudiantes por parte de la AME.
Previo a iniciar el proyecto, se realizaron dos visitas a Cayambe para mantener reuniones de planificación con las autoridades ambientales del GADIP y el Presidente de la Junta de Agua de La Tola y formalizar la presentación del proyecto. Además se confirma que el agua consumida proviene de fuentes subterráneas del volcán Cayambe y captada en un pozo, de donde es bombeada a una reserva alta para luego distribuirla por gravedad mediante mangueras sin ningún cuidado.

En el arranque del Proyecto se presentaron formalmente docentes, representantes de la AME y estudiantes, señalando que la próxima visita será para levantar encuestas y elaborar la línea base.

Posterior, en otra visita, se diseñó y aplicó para el estudio inductivo-deductivo la encuesta, puerta a puerta, que recopiló la información que generó el informe técnico de la línea base y la toma de muestras a lo largo de la línea de agua, correspondiendo:

1. La primera toma en la salida del pozo, M2.

Zona: $17 \mathrm{~N}$.

Latitud: $8116.00 \mathrm{~m}$. N.

Longitud: $817094.00 \mathrm{~m}$. E.

Altura: $2804 \mathrm{~m}$.

2. La segunda toma en el Pozo de agua, M2.

Zona: $17 \mathrm{~N}$.

Latitud: $8116.00 \mathrm{~m} . \mathrm{N}$.

Longitud: $817094.00 \mathrm{~m}$.

Altura 2804.

3. Tercera toma al inicio de la comunidad, M3. Zona: $17 \mathrm{~N}$.

Latitud: $7597.00 \mathrm{~m}$. N.

Longitud: $816179.00 \mathrm{~m}$. E.

Altura $2792 \mathrm{~m}$.

4. La cuarta toma en válvula de desagüe (final de la comunidad) M1.

Zona: $17 \mathrm{~N}$

Latitud: $6398.00 \mathrm{~m} . \mathrm{N}$.

Longitud: $815863.00 \mathrm{~m} . \mathrm{N}$

Altura: $2774 \mathrm{~m}$. 
Las tomas M2 se ubican en el mismo punto, lo señalado está respaldado con registro de fotos georreferenciadas del sector y los sitios donde se efectuó la captación de las muestras, aplicando los protocolos establecidos, para su transporte y destino a un laboratorio particular especializado que efectuó los análisis físico-químicos y bactereológico; originando los primeros resultados que generó la línea base para formular la estrategia de intervención fundamentalmente en capacitación, seguimiento y evaluación.

En otra visita los docentes informan a las autoridades del Gobierno Seccional los resultados de las encuestas, de los análisis físico-químico y bactereológico del agua, para formalizar entre el Alcalde del GADIP y Rector de la UTE, un Convenio Específico de Cooperación Interinstitucional, bajo el compromiso de "(...) contribuir al conocimiento de una alternativa para purificar el agua con el fin de mejorar la calidad de vida de las personas en la comunidad La Tola del cantón Cayambe", además se firma el Convenio donde la A.M.E., apoya técnicamente para lograr los objetivos planteados en el Proyecto original.

Se socializan los resultados de las encuestas a la Comunidad, la línea base y la solución para contar con agua purificada en las casas, así como elevar sugerencias al Municipio de Cayambe.

Se entrega la Guía Didáctica y materiales para aplicar el método SODIS en todas las viviendas también los recipientes a utilizar, llenan el registro y se explica el método de la siguiente manera:

- Pintar un lado del recipiente de tres litros con color negro.

- Llenarlo de agua al tope, de manera que al cerrarlo no permita el ingreso de aire.

- Ubicar el recipiente con agua horizontalmente en un lugar donde pueda recibir la radiación solar, es importante que la parte pintada vaya sobre la superficie a colocar.

La quinta visita es para receptar muestras del agua, una vez aplicado el método SODIS, analizadas en el Laboratorio de Agua y Suelo, según la normativa para agua INEN 1108, y determinar la presencia de: coliformes fecales, para criptospori- dium y para giardia lambia. El resultado contó con la conformidad del Evaluador de la Facultad de Ciencias de la Salud sobre la técnica aplicada y cumplimiento descrito en el "Informe Técnico". La AME avala la metodología aplicada. Ante los dirigentes comunitarios, docentes y estudiantes se socializan los resultados alcanzados. A fin de motivar su uso, se entregan promocionales, como camisetas con los logotipos de la UTE y SODIS a cada representante de familia en la Casa Comunal de La Tola, de forma que se pueda multiplicar el uso y beneficios del método SODIS. Simultáneamente se pudo constatar que alrededor del $50 \%$ de las familias, lo estaban aplicando.

\section{Resultados}

La implementación del método SODIS patrocinado por la UTE beneficia a 300 personas, bajo el Programa de Vinculación 'Conservación y Aprovechamiento Sostenible de los Recursos Naturales' en el Área de conocimientos orientada al Agua en la Sección Sexta del Capítulo Segundo del Título VII Régimen del Buen Vivir (Constitución 2008).

La primera asignación económica inició operativamente el Proyecto planificado y administrativamente aprobado. Así en la segunda visita, se tomó la primera muestra de agua, que arrojó, en el Laboratorio TRAHISA, resultados del análisis físicoquímico y bactereológico muy similares en los tres puntos muestreados (M1, M2 y M3), destacando los resultados que salen del rango previsto:

TABlA 1

\begin{tabular}{l|c|c}
\hline Parámetro & Límite tolerable & Resultado \\
\hline Color & 15.0 & 7.50 \\
\hline Turbiedad & 5.0 & 7.77 \\
\hline Flúor & 1.5 & 0.30 \\
\hline Manganeso & 0.4 & 0.00 \\
\hline Nitratos & 50.0 & 1.32 \\
\hline Nitritos & 0.2 & 0.00 \\
\hline $\begin{array}{l}\text { Coliformes } \\
\text { fecales }\end{array}$ & $<1.1$ & $<1.1$ \\
\hline
\end{tabular}

Elaborada por: los autores 
TABLA 2

\section{Resultados del análisis}

No satisface las normas físico-químicas

de potabilidad del agua

\begin{tabular}{ll}
\hline Concentración elevada de hierro & Presentan problemas estéticos \\
\hline Concentración elevada de nitratos & $\begin{array}{l}\text { Riesgo en los recién nacidos de adquirir } \\
\text { methahemoglobinemia infantil }\end{array}$ \\
\hline Concentración elevada de cloruros y de alcalinidad & Un sabor salado en el agua \\
\hline Concentración elevada de sulfatos & Perturbaciones gastrointestinales \\
\hline Concentración elevada de dureza & $\begin{array}{l}\text { Incrustaciones en tuberías y dificulta } \\
\text { la disolución en jabones }\end{array}$ \\
\hline Concentraciones elevadas de manganeso & Alteraciones estéticas \\
\hline Concentraciones elevadas de fosfatos & Crecimiento de algas y plantas \\
\hline
\end{tabular}

- Satisface las normas microbiológicas de potabilidad de agua establecidas

- Existe contaminación de tipo microbiológico, se recomienda inspección, protección y aumento del nivel de cloro en el sistema de distribución y después se deben realizar nuevos análisis.

- Agua contaminada no apta para consumo humano

- Debido al elevado número de bacterias se recomienda inspección, protección y desinfección

- El agua es incrustante.

- El agua es corrosiva.

Elaborada por: los autores

Se destacó:

Análisis físico-químico.- Alta presencia de hierro, haciéndola no apta para el consumo, especialmente de niños, donde se debe insistir en el objetivo de capacitar a la comunidad en el uso adecuado del agua.

Análisis Biológico.- Se determinó la presencia de: coliformes totales y fecales Escherichia Coli, según el método de análisis de recuento externo de placas petrifilm, se pudo establecer que el resultado es $0 \mathrm{UFC} / \mathrm{g}$ de muestra. Adicionalmente el examen microbiológico señaló que se satisfacían las normas microbiológicas de potabilidad de agua establecida, contando con un aumento del nivel de cloro y un nuevo monitoreo para determinar los resultados.
La encuesta, entregó las siguientes conclusiones:

a. La mayoría de la Comunidad se considera mestiza; su principal ocupación es la agricultura, a la que se dedican los hombres. Las mujeres tienen como actividad principal los quehaceres domésticos.

b. Con respecto a las enfermedades de tipo hídrico, la mayoría de niños tienen parasitosis, seguido de las enfermedades respiratorias y en menor cantidad las enfermedades infecciosas de piel. Alrededor del $80 \%$ de encuestados afirmaron que sí conocían el origen de las enfermedades, especialmente de la diarrea y sabían cómo curarla.

c. Al enfermar las personas acudían al Hospital de Cayambe, ya que el Centro de Salud en 
la parroquia de Ayora, que es más cercano, se hacía más difícil llegar por falta de una vía de acceso, especialmente cuando de emergencias se trataba. La consulta tenía un costo promedio 10 dólares.

d. El 51\% de la población utilizaba agua de la red pública, el resto se abastece del pozo y agua del río. El 26\% acarreaba agua del río para las necesidades diferentes a las alimentarias, esta actividad la realizaban todos los miembros de la familia.

e. Había insatisfacción con el servicio que recibían, al no disponer de medidor de consumo, la opción de comprar agua embotellada no era aceptada, frente al pago de 5 dólares mensuales que realizaban al GADIP por el servicio.

f. Para la disposición de excretas y basura, el 65\% de las familias contaban con red de alcantarillado, el 26\% usa fosa séptica y el resto letrina.

g. El sistema de recolección de residuos sólidos llegaba al $74 \%$ de la población. Cuando no había recolección, quemaban la basura o la arrojaban al río.

h. La familia almacenaba agua dentro de la casa en recipientes con tapa y hervían el agua para beber.

i. El 77\% se lavaban las manos antes de comer y el mismo porcentaje lavaba los alimentos.

j. El 78\% se lavaba las manos después de hacer sus evacuaciones y mantenían a sus animales cerca de las casas.

k. La comunidad si había recibido capacitación de cómo se administra el agua, pero no aplicaban los conocimientos aprendidos.

Luego de tres meses se tomó otra muestra, esta vez del agua, aplicado el método SODIS, en los puntos donde se lo hizo la primera vez, y es en el Laboratorio de Agua y Suelo donde se comparó con el resultado del análisis físico-químico de TRAHISA destacando los parámetros que no cumplían con la Normativa:

Tabla 3. ANÁlisis FÍsico-QUímico del AgUA

\begin{tabular}{c|c|c|c|c}
\hline Muest. & Parámet. & Límite $\mathbf{m a x}$ & Resultado & Observac. \\
\hline M2 & turbiedad & 5,0 & 7,7 & No cumple \\
\hline M3 & turbiedad & 5,0 & 50,7 & No cumple \\
\hline M2 & Hierro total & 2,26 & 1 & No cumple \\
\hline M3 & Hierro total & 1,16 & 1,00 & No cumple \\
\hline
\end{tabular}

Elaborada por: Los autores

Por el análisis las muestras cumplen con la normativa INEN 1108 para Calidad de Agua Potable, excepto las muestras M2 y M3, en el ítem turbiedad. Ambas en el punto Casa Comunal. Los resultados del análisis, con respecto a la normativa ambiental del Texto Unificado de Legislación Ambiental Secundaria, Libro VI, anexo 1, indican que, la muestra no cumplía con el límite máximo permisible de concentración de hierro total.

\section{Análisis Microbiológico del Agua}

Igualmente se realizaron en los dos laboratorios, TRAHISA, y el segundo de Agua y Suelo de la UTE aplicando la normativa 1108. Se utilizó el método de Tubos múltiples NMP/100 ml. para coliformes fecales; para cryposporidium, número de quistes/100 litros y para giardia, número de quistes/100 litros. 
Se resumen los resultados obtenidos por seis evaluadores en dos momentos, sin aplicar el método SODIS y con el método SODIS:

TABLA 4

\begin{tabular}{c|c|c|c|c}
\hline Origen & Parámetro & Sin SODIS & Con SODIS & Observación \\
\cline { 1 - 4 } Fin de tubería & Coliformes fecales & Ausencia & Ausencia & Cumple \\
\hline Fin de tubería & Cryptospo-dium & Presencia & Ausencia & Cumple \\
\hline Fin de tubería & Giardia & Presencia & Ausencia & Cumple \\
\hline
\end{tabular}

Se determinó que el método SODIS es efectivo para el control de los microorganismos.

El Informe Técnico fue puesto a consideración de la Facultad de Ciencias de la Salud "Eugenio Espejo", de la AME y de la Evaluadora del Proyecto, quienes otorgaron sus avales.

Para el cierre del Proyecto se convocó a los dirigentes de La Tola, que fueron informados sobre lo desarrollado y se firmaron las cartas de haber sido beneficiados por la socialización del método SODIS. Sugieren que se replique en otras comunidades del GADIP de Cayambe.

En la última visita a la Comunidad, se constató que alrededor del $50 \%$ de las familias aplicaban de manera regular el método SODIS.

A la clausura del Proyecto, se utilizaron USD. 2.060,00 de los USD. 3.450,00 presupuestados.

\section{Productos}

Implica lograr el $100 \%$ en cuatro productos con indicadores cumplidos, verificados y finalmente evidenciados.

\section{Producto 1}

Diagnóstico en la Comunidad.

Indicador.- Se elaboró el diagnóstico de la calidad del agua que se consumía.
Medios de verificación.- Respaldo con evidencias

a. Diagnóstico de la calidad del agua de la comunidad La Tola.

b. Aval técnico de la AME.

c. Aval de la Facultad de Ciencias de la Salud.

Estado.- Logrado el 100\%.

\section{Producto 2}

Guía de uso del método SODIS.

Indicador.- Guía de Información para su aplicación.

Medios de verificación.-

a. Guía impresa del método

b. Aval de los técnicos de la AME.

c. Aval de la Facultad de Ciencias de la Salud de la UTE.

Estado.- Logrado 100\%.

\section{Producto 3}

Socialización y capacitación a los moradores sobre la aplicación e importancia del uso del método.

Indicador.- $\mathrm{Al}$ quinto mes se difundió y capacitó al $80 \%$ de los moradores, de los cuales alrededor del $50 \%$ de las familias capacitadas lo utilizaban en sus hogares.

Medios de verificación.- Se respalda con:

a. Fotografías de reuniones.

b. Hojas de asistencias.

c. Levantamiento de la información sobre el uso del método en los hogares.

d. Aval de la AME.

e. Acta de recepción por parte de la Comunidad. Estado.- logrado 100\%. 


\section{Producto 4}

Informe Final con lo actuado y resultados alcanzados aplicando el método SODIS.

Indicador.- Informe Final.

Medios de verificación.- Informe Final físico y en digital con:

a. Convenio UTE y GADIP de Cayambe.

b. Informe Técnico de Avance

c. Resultados de la aplicación del método SODIS avalado por la AME y Facultad de Ciencias de la Salud.

d. Informe Financiero

e. Respaldo Financiero

f. Pedido de certificaciones para docentes y estudiantes.

g. Acta de entrega de promocionales (camisetas) a participantes de la Comunidad.

\section{Conclusiones}

- El método SODIS se fundamenta en aprovechar la radiación solar (rayos UV) y el aumento de la temperatura del agua, para inactivar y destruir los organismos patógenos presentes en el agua, desinfectándola" (ficha tecnológica 10, Método SODIS para purificación del agua. SERIE III: FICHAS TECNOLÓGICAS).
- El método funciona eficazmente en una exposición de 6 horas bajo radiación solar de al menos $500 \mathrm{~W} / \mathrm{m}^{2}$.

- Se detectaron tanques de almacenamiento de agua en malas condiciones y sin recubrimiento de protección.

- Es relevante para las autoridades y población, contar con información acerca del estado del agua que consumían y los beneficios de aplicar el método SODIS.

- La cantidad de hierro en un punto al final de la distribución, no cumplía con los criterios establecidos en el Texto Unificado de Legislación Ambiental Secundaria, por lo que no era apta para el consumo humano, especialmente de niños.

- El análisis biológico para determinar la presencia de: coliformes totales y fecales, escherichia coli, según el método de análisis de recuento externo de placas petrifilm, estableció que el resultado fue de $0 \mathrm{UFC} / \mathrm{g}$ de muestra.

- Se concluye que el método SODIS es efectivo para el control y eliminación de los microorganismos como cryptosporidium y giardia, detectados en la primera muestra.

\section{Referencias bibliográficas}

- CEPAL (2012). Diagnóstico de la información estadísticadelaguaenEcuador.Informefinal.Recuperado de <http://aplicaciones.senagua.gob.ec/ servicios/descargas/archivos/download/Diagnostico $\% 20$ de $\% 20$ las\%20Estadisticas\%20del\%20 Agua\%20Producto\%20IIIc\%202012-2.pdf>

- Asamblea Nacional. Constitución de la República del Ecuador, 2008. Recuperado de: <http:// www.asambleanacional.gov.ec/documentos/ constitucion_de_bolsillo.pdf>
- COSUDE, Agencia Suiza para el Desarrollo y la Cooperación, S. F. (2003). SODIS, Desinfección Solar del Agua. Programa de Agua y Saneamiento Región América Latina y el Caribe, Reporte SANDEC No. 07/03. Recuperado de: $<$ http://www.sodis.ch/methode/anwendung/ ausbildungsmaterial/dokumente_material/ manual_s.pdf>

- CEPIS/OPS. ADRA. COSUDE. Desinfección Solar del Agua. Proyecto demostrativo. 
- Desinfección solar (SODIS), un método alternativo para el tratamiento de agua de consumo humano Recuperado de: <http://www.cnea.gov. ar/xxi/ambiental/agua-pura/prreesentacionesencuentro/ARGENTINA\%20SODIS.pdfs

- Fundación SODIS (2013). Desinfección Solar del Agua. En F. d. Simón, Servicio de Linchtenstein para el Desarrollo. Cochapamba- Bolivia.

- Naciones Unidas, (2016). Agua y empleo. Recuperado de: <http://unesdoc.unesco.org/ images/0024/002441/244103s.pdf>

- Reforma del Libro VI del Texto Unificado De Legislación Secundaria. (2015). Registro Ofi- cial, Órgano del Gobierno del Ecuador. Quito: Ministerio del Ambiente.

- Registro Oficial No 305 Segundo Suplemento, Ley Orgánica De Recursos Hídricos, Usos y Aprovechamiento del Agua 6 de Agosto de 2014.

- SODIS, Desinfección Solar del Agua. Guía de aplicación. PAS, EAWAG-SANDEC. SODIS, UNICEF. COSUDE.

- s/a. Agua e hidratación. Recuperado de: $<$ http://www.h4hinitiative.com/es/ciencia-dela-hidratacion/laboratorio-de-hidratacion/hidratacion-para-los-adultos/equilibrio-hidrico> 